\title{
Microbial Phytase and Nutrient Utilization in Low Phosphorus Chicken Diets
}

\author{
Frank Liebert, John K. Htoo and Angela Sünder \\ Institute for Animal Physiology and Animal Nutrition, Georg-August-University, Kellnerweg 6, 37077 Goettingen, Germany
}

\begin{abstract}
Experiments were conducted with male growing chicken (Cobb 500) to examine effects of added microbial phytase on growth performance and nutrient utilization in corn-soybean meal (CSM) and wheat-soybean meal (WSM) diets. Low P basal diets (CSM1: 0.15 / 0.11\% NPP in DM of starter/grower diets; WSM1: 0.16/0.12\% NPP in DM of starter/grower diets) were supplemented with 250 or $500 \mathrm{U} / \mathrm{kg}$ of an experimental microbial phytase (CSM2, CSM3; WSM2, WSM3) or 1.5 $\mathrm{g} / \mathrm{kg}$ inorganic phosphorus (CSM4, WSM4). Growth studies were conducted (7-42 d) to establish nutrient deposition (6 pens per diet, 10 birds per pen). Separate balance studies $(n=8)$ yielded individual $\mathrm{N}$ and $\mathrm{P}$ balance data for the age period 21-25 d. Additionally, studies with CSM diets also provided tibia composition data.

Phytase supplementation in CSM diets yielded significant effects on growth and deposition data. Additionally, the mortality rate declined significantly and tibia composition (ash, P, Ca) was improved. WSM diets provided more pronounced effects on deposition data, but growth responded only numerical. Balance studies delivered significant effects on P utilization due to phytase addition in both CSM and WSM diets, respectively. However, lower P utilization was observed in CSM diets. Significant effects of phytase addition on $\mathrm{N}$ utilization were established only in CSM diets. Consequently, generalized conclusions due to improved $\mathrm{N}$ utilization following phytase addition are not supported by the current study.
\end{abstract}

Abbreviation key: $\mathrm{CSM}=$ corn-soybean meal; iP $=$ inorganic $\mathrm{P} ; \mathrm{NPP}=$ non-phytate $\mathrm{P} ; \mathrm{WSM}=$ wheat-soybean meal

Key words: growing chicken, growth performance, nutrient utilization, phytase, $\mathrm{P}$ utilization

\section{Introduction}

Lack of endogenous phytase activity limits phytate degradation in the gut of growing chicken (Nelson, 1967; Davies et al., 1970). Improved $\mathrm{P}$ utilization is achieved following supplementation of microbial phytase, as reported extensively (Simons et al., 1990; Kornegay, 2001; Shirley and Edwards, 2002; Augspurger and Baker, 2004; Snow et al., 2004; Payne et al., 2005; Ebrahimnezhad et al., 2008). Contradictory reports are published in laying hens (Peter et al., 1992; van der Klis et al., 1997; Carlos and Edwards, 1998; Boling et al., 1997, 2000a, b; Gordon and Roland, 1997, 1998; Rama Rao et al., 1999; Jalal and Scheideler, 2001; Shan et al., 2002; Ceylan et al., 2003; Keshavarz, 2003a, b; Lim et al., 2003; Liebert et al., 2005). Different sources of microbial phytase may also affect phytate degradation (Augspurger et al., 2003; Zyla et al., 2004; Liebert and Portz, 2005, 2007). Furthermore, effects of added phytase on nutrient utilization in growing chicken are possible. Phytate-

Received: March 25, 2008, Accepted: May 20, 2008

Correspondence: Prof. Dr. F. Liebert, Institute for Animal Physiology and Animal Nutrition, Kellnerweg 6, 37077 Goettingen, Germany. (E-mail: flieber@gwdg.de) protein-bindings in the gut (O'Dell and De Boland, 1976; Gifford and Clydesdale, 1990) could play a significant role and effects of phytates on endogenous amino acid losses are reported (Cowieson and Ravindran, 2007). Consequently, improved phytate hydrolysis could yield enhanced protein digestibility. Inhibiting effects of phytates on several enzyme activities were mostly observed in vitro (Deshpande and Cheryan, 1984; Knuckles et al., 1989; Caldwell, 1992). Marginal effects on improvement of digestibility were expected in vivo (Reddy et al., 1988; Vaintraub and Bulmaga, 1991; Zhang et al., 1999). According to several reports (Sebastian et al., 1997; Zhang et al., 1999; Peter et al., 2000; Augspurger and Baker, 2004), Bedford (2000) concluded inconsistent secondary effects of microbial phytase in growing chickens. In contrast, other studies confirmed significant responses (Farrell et al., 1993; Yi et al., 1994, 1996a; Kornegay et al., 1996; Sebastian et al., 1996; Namkung and Leeson, 1999; Zhang et al., 1999; Lan et al., 2002; Juanpere et al., 2004; Rutherford et al., 2004; Onyango et al., 2005). Several factors could be of importance, like type of applied microbial phytase, solubility of phytates, and native phytase activity. Dietary calcium supply is an additional factor (Plumstead et al., 2008).

The present study was conducted to examine effects of 
an experimental microbial phytase in low $\mathrm{P}$ corn-soybean meal (CSM) and wheat-soybean meal (WSM) on growth, feed efficiency and nutrient utilization in growing chicken.

\section{Materials and Methods}

\section{Animals and Housing}

Day old male growing chickens (Cobb 500) from a commercial hatchery reared under controlled climate conditions in a cage system. Room temperature during rearing started with $32^{\circ} \mathrm{C}$ in the first week after hatching and declined by $2^{\circ} \mathrm{C}$ per week, lighting was 24 hours per day. Chicken were fed a standardized starter diet based on wheat and soybean meal (DM based: $266 \mathrm{~g} \mathrm{~kg}^{-1}$ crude protein, $13 \mathrm{MJ} \mathrm{kg}^{-1} \mathrm{AMEn}$ ) on free choice level up to six days. Two experiments were conducted, designed as growth trial (7-42d) and balance study (21-25d) using separate birds:

Experiment 1 utilized low $\mathrm{P}$ corn-soybean (CSM) diets (Table 1) with low native phytase activity, two steps of microbial phytase and a control diet with inorganic $\mathbf{P}$ addition (Table 2).

Experiment 2 examined low $\mathrm{P}$ wheat-soybean (WSM) diets (Table 1) with enhanced dietary native phytase activity from wheat, two steps of microbial phytase and a control diet with inorganic $\mathrm{P}$ addition (Table 2).

As an example for low and high native phytase activity in the diet, the experiments utilized CSM and WSM diets, respectively. Each growth experiment was conducted as randomized design using totally 240 male chickens in 24 floor pens (average stocking density 7.6 chickens $\mathrm{m}^{-2}$ ), short cut straw bedding, controlled climate conditions and a continuous lighting regime, respectively. Diets were randomly assigned to six pens (10 birds per pen). Growth experiments were divided into starter (7-20 d) and grower period (21-42d) with free choice level of feed and water supply.

Balance studies with individual metabolism cages $(n=$ 8 ) utilized an adaptation period (16-20d) and a period of quantitative excreta collection (21-25d). Individual feeding adapted to body weight minimized feed losses and contamination of excreta by feed particles.

The experiments were carried out at the facilities of the Institute for Animal Physiology and Animal Nutrition in accordance with animal welfare legislations and were approved by the Ethics Committee of the Agricultural Faculty of Goettingen University.

\section{Experimental Diets}

Low $\mathbf{P}$ negative control diets (CSM1, WSM1) and

Table 1. Composition of the basal CSM and WSM diets

\begin{tabular}{|c|c|c|c|c|}
\hline \multirow{2}{*}{$\frac{\text { Diets }}{\text { Ingredients }(\mathrm{g} / \mathrm{kg})}$} & \multicolumn{2}{|c|}{ Corn-soybean meal (CSM) } & \multicolumn{2}{|c|}{ Wheat-soybean meal (WSM) } \\
\hline & Starter & Grower & Starter & Grower \\
\hline Corn & 449 & 538 & - & - \\
\hline Wheat & - & - & 462 & 549 \\
\hline Soybean meal & 445 & 370 & 423 & 345 \\
\hline Soybean oil & 33 & 20 & 43.5 & 35 \\
\hline Wheat starch & 36 & 36 & 36 & 36 \\
\hline Calcium carbonate & 22 & 22 & 21 & 22 \\
\hline Salt & 5 & 4 & 4.5 & 3 \\
\hline Premix $^{1)}$ & 10 & 10 & 10 & 10 \\
\hline \multicolumn{5}{|l|}{ Analyzed (DM\%) } \\
\hline Crude protein & 26.5 & 24.1 & 26.9 & 23.8 \\
\hline Crude fat & 7.4 & 5.9 & 6.8 & 5.5 \\
\hline Crude fiber & 4.9 & 4.7 & 4.0 & 3.9 \\
\hline Crude ash & 6.8 & 6.8 & 6.9 & 6.5 \\
\hline Starch & 37.2 & 40.3 & 34.4 & 41.3 \\
\hline Sugar & 5.9 & 6.6 & 6.7 & 6.3 \\
\hline Lysine & 1.52 & 1.32 & 1.51 & 1.31 \\
\hline Met + Cys & 1.05 & 0.99 & 1.05 & 0.99 \\
\hline Threonine & 1.04 & 0.94 & 1.01 & 0.90 \\
\hline Total phosphorus & 0.46 & 0.43 & 0.46 & 0.42 \\
\hline Non-phytate phosphorus & 0.15 & 0.11 & 0.16 & 0.12 \\
\hline Calcium & 1.28 & 1.14 & 1.23 & 1.24 \\
\hline $\mathrm{AME}_{\mathrm{n}}\left(\mathrm{MJ} / \mathrm{kg} \mathrm{DM}{ }^{2}\right)$ & 13.6 & 13.3 & 12.4 & 12.8 \\
\hline \multicolumn{5}{|c|}{$\begin{array}{l}\text { 1) Provided (per kilogram of diet): vitamin } \mathrm{A}, 5000 \mathrm{IU} \text {; vitamin } \mathrm{D}_{3}, 1000 \mathrm{IU} \text {; vitamin } \mathrm{E}, 30 \mathrm{mg} \text {; } \\
\text { vitamin } \mathrm{B}_{1}, 2.6 \mathrm{mg} \text {; vitamin } \mathrm{B}_{2}, 4.8 \mathrm{mg} \text {; vitamin } \mathrm{B}_{6}, 3.2 \mathrm{mg} \text {; vitamin } \mathrm{B}_{12}, 20 \mathrm{mcg} \text {; vitamin } \mathrm{K}_{3}, 3 \\
\mathrm{mg} \text {; nicotinic acid, } 50 \mathrm{mg} \text {; calcium pantothenate, } 10 \mathrm{mg} \text {; folic acid, } 0.9 \mathrm{mg} \text {; biotin, } 100 \mathrm{mg} \text {; } \\
\text { choline chloride, } 1000 \mathrm{mg} \text {; Se, } 25 \mathrm{mg} \text {; } \mathrm{Zn}, 80 \mathrm{mg} \text {; } \mathrm{Mn}, 120 \mathrm{mg} \text {; Cu, } 30 \mathrm{mg}, \mathrm{Fe}, 50 \mathrm{mg} ; \mathrm{I}, 1.2 \mathrm{mg} \text {; } \\
\text { Co, } 0.55 \mathrm{mg} \text {; Monensin-Na } 125 \mathrm{mg} \text {; and BHT, } 100 \mathrm{mg} \text {. } \\
\text { 2) N-corrected apparent metabolizable energy, calculated (WPSA, 1984). }\end{array}$} \\
\hline
\end{tabular}


Table 2. Experimental design

\begin{tabular}{|c|c|c|c|c|c|c|c|c|}
\hline \multirow{2}{*}{$\begin{array}{c}\text { Experiment } \\
\text { Diets } \\
\text { Supplementation }\end{array}$} & \multicolumn{4}{|c|}{$\begin{array}{c}1 \\
\text { Corn-soybean meal (CSM) }\end{array}$} & \multicolumn{4}{|c|}{$\begin{array}{c}2 \\
\text { Wheat-soybean meal (WSM) }\end{array}$} \\
\hline & CSM1 & CSM2 & CSM3 & CSM4 & WSM1 & WSM2 & WSM3 & WSM4 \\
\hline Phytase $(\mathrm{U} / \mathrm{kg} \text { diet })^{1}$ & 0 & 250 & 500 & 0 & 0 & 250 & 500 & 0 \\
\hline Inorganic $\mathbf{P}(\mathrm{g} / \mathrm{kg} \text { diet })^{2}$ & 0 & 0 & 0 & 1.5 & 0 & 0 & 0 & 1.5 \\
\hline Phytase activity $(\mathrm{U} / \mathrm{kg})^{3}$ & 56 & 230 & 421 & 61 & 448 & 762 & 956 & 458 \\
\hline
\end{tabular}

positive control diets (CSM4, WSM4) supplemented with inorganic $\mathrm{P}$ (Monosodium phosphate) were prepared (Table 2). Nutrient composition (Table 1) ensured or exceeded recommendations of NRC (1994), except P supply $(0.44 \%$ total $\mathrm{P}$ and $0.13 \% \mathrm{NPP})$. Two steps of microbial phytase (250 and $500 \mathrm{U} / \mathrm{kg}$ ) were investigated (CSM2, CSM3 and WSM2, WSM3) without supplementation of inorganic P. Diets were pelleted under controlled mild conditions, temperatures near to the outlet of the $2 \mathrm{~mm}$ die did not exceed $50^{\circ} \mathrm{C}$. The examined phytase SP 1002 (Former Roche Vitamins Ltd, Basel, Switzerland) was an experimental Consensus phytase produced by a genetically modified strain of the yeast Hansenula polymorpha. The production strain is carrying a synthetic gene coding for 3-phytase (IUB no. 3.1.3.8.) with elevated $\mathrm{pH}$ optimum $(>5)$ and increased stability against thermal treatment and proteolytic activity.

\section{Measurements}

\section{Growth Experiments}

Growth and feed intake were weekly recorded for each pen. At the end of the experiment (42d), for each diet totally six imposing birds for body weight (one bird per pen) were sampled for individual analysis of body nutrient composition. A pooled sample of five birds with average body weight was utilized for body analyses at the beginning of the growth experiments. Additionally, in experiment 1 the left tibia bone from six birds per diet was prepared. Following removal of adhering tissue, drying $\left(105^{\circ} \mathrm{C}, 48 \mathrm{~h}\right)$ and overnight ashing at $550^{\circ} \mathrm{C}$, crude ash, $\mathrm{Ca}$ and $\mathrm{P}$ were analyzed.

\section{Balance Experiments}

Following the adaptation period (16-20 d), a collection period $(21-25 d)$ was conducted with quantitative excreta collection three times a day. Total excreta samples kept frozen at $-18^{\circ} \mathrm{C}$ until further analysis. $\mathrm{N}$ utilization parameters were calculated from difference between $\mathrm{N}$ intake and total $\mathrm{N}$ excretion ( $=\mathrm{N}$ balance) related to the total $\mathrm{N}$ intake. $\mathrm{P}$ utilization was calculated accordingly. $\mathrm{N}$ digestibility was calculated according to the $\alpha$-amino- $\mathrm{N}$ procedure (Pahle et al., 1985) assuming that $\mathrm{N}$ excretion via feces is mostly $\alpha$-amino-N. The energy content of the experimental diets $\left(\mathrm{AME}_{\mathrm{n}}\right)$ was determined by calorimetric measurements in feed and excreta, including $\mathbf{N}$ corrections for individual $\mathrm{N}$ deposition (Titus et al., 1959).

\section{Chemical Analysis}

Gross energy (AC 350 LECO Instruments) and N (Dumas method, FP 2000 LECO Instruments) were determined in the experimental diets and freeze-dried excreta samples. Total $\mathbf{P}$ in feed, excreta and tibia ash analyses run according to Naumann and Bassler (1997). In feed and tibia samples, $\mathrm{Ca}$ was determined according to Naumann and Bassler (1997) using an atomic absorption spectrophotometer (SpectrAA 55, Varian). Analysis of phytate $\mathbf{P}$ in feed samples was according to Association of Official Analytical Chemists (1990). The $\alpha$-amino-N in feed and excreta samples was analyzed in line with the procedure of Pahle et al. (1983, 1985). In-feed activity of phytase was determined due to Engelen et al. (1994).

\section{Statistical Analysis}

Results are presented as means \pm standard deviation ( \pm $\mathrm{SD})$. Experimental unit was the pen (growth studies) and the individual bird (balance experiments), respectively. Data analysis in growth studies was based on one way ANOVA using Fisher-LSD or Games-Howell post-hoc test according to equality or non-equality of variances (verified by Levene-test). Data analysis of the balance experiments utilized univariate ANOVA. Univariate data analysis was utilized for balance studies to demonstrate possible effects of the basal diets under study on metabolic parameters. Under circumstance of standardized balance experiments, it could be assumed that the possible effect was lower compared to the growth studies.

\section{Results}

\section{Growth Data}

CSM diets (Table 3) demonstrate significant effects of iP supplementation (CSM1 vs. CSM4) on zootechnical data and realize the marginal $P$ supply in diet CSM1 in correspondence with very low native phytase activity (Table 2). Both factors were responsible for the observed increase of mortality (CSM1: 18.3\%; CSM2: 11.7\%; CSM 3: 3.3\%; CSM 4: 1.7\%; WSM 1: 16.7\%; WSM 2: 13.3\%; WSM3: 5\%; WSM4: 0). Provided FCR data were not significantly affected. Adding phytase yielded significant dose depending response on growth data and feed intake, but insignificant effects on FCR. In addition, 
Table 3. Growth performance of chicken fed low phosphorus CSM diets (7-42 d) supplemented with microbial phytase or inorganic phosphorus and results of ANOVA, Experiment 1 (6 pens, 10 birds per pen)

\begin{tabular}{|c|c|c|c|c|c|c|c|}
\hline \multirow{2}{*}{ Diets } & \multicolumn{2}{|c|}{ Supplementation } & \multirow{2}{*}{$\begin{array}{l}\text { Initial body weight } \\
\qquad \mathrm{d} 7\end{array}$} & \multirow{2}{*}{$\begin{array}{l}\text { Final body weight } \\
\text { d42 }\end{array}$} & \multirow{2}{*}{$\begin{array}{l}\text { Feed } \\
\text { intake }\end{array}$} & \multirow{2}{*}{$\begin{array}{l}\text { Body } \\
\text { weight gain }\end{array}$} & \multirow{2}{*}{ FCR } \\
\hline & Phytase & iP & & & & & \\
\hline & $(\mathrm{U} / \mathrm{kg})$ & $(\mathrm{g} / \mathrm{kg})$ & (g) & (g) & $(g / d)$ & $(g / d)$ & $(g / g)$ \\
\hline CSM1 & 0 & 0 & $142.9 \pm 0.5$ & $1362.9^{a} \pm 48.8$ & $70.6^{\mathrm{a}} \pm 3.2$ & $34.9^{\mathrm{a}} \pm 1.4$ & $1.99 \pm 0.1$ \\
\hline CSM2 & 250 & 0 & $142.5 \pm 0.6$ & $1579.0^{\mathrm{b}} \pm 43.6$ & $81.6^{\mathrm{b}} \pm 3.3$ & $41.0^{\mathrm{b}} \pm 1.3$ & $1.94 \pm 0.1$ \\
\hline CSM3 & 500 & 0 & $142.5 \pm 0.7$ & $1830.3^{\mathrm{c}} \pm 74.2$ & $92.8^{c} \pm 3.9$ & $48.2^{\mathrm{c}} \pm 2.1$ & $1.87 \pm 0.0$ \\
\hline CSM4 & 0 & 1.5 & $142.8 \pm 0.8$ & $2131.8^{\mathrm{d}} \pm 54.3$ & $116.0^{\mathrm{d}} \pm 11.9$ & $56.8^{\mathrm{d}} \pm 1.6$ & $1.97 \pm 0.2$ \\
\hline
\end{tabular}

${ }^{\mathrm{a}, \mathrm{b}}$ Means within the same column with different superscript letters are significantly different $(p<0.05)$.

Table 4. Growth performance of chicken fed low phosphorus WSM diets (7-42 d) supplemented with microbial phytase or inorganic phosphorus and results of ANOVA, Experiment 2 (6 pens, 10 birds per pen)

\begin{tabular}{|c|c|c|c|c|c|c|c|}
\hline \multirow{2}{*}{ Diets } & \multicolumn{2}{|c|}{ Supplementation } & \multirow{2}{*}{$\begin{array}{l}\text { Initial body weight } \\
\qquad \mathrm{d} 7\end{array}$} & \multirow{2}{*}{$\begin{array}{l}\text { Final body weight } \\
\text { d42 }\end{array}$} & \multirow{2}{*}{$\begin{array}{l}\text { Feed } \\
\text { intake }\end{array}$} & \multirow{2}{*}{$\begin{array}{c}\text { Body } \\
\text { weight gain }\end{array}$} & \multirow{2}{*}{ FCR } \\
\hline & Phytase & iP & & & & & \\
\hline & $(\mathrm{U} / \mathrm{kg})$ & $(\mathrm{g} / \mathrm{kg})$ & (g) & (g) & $(g / d)$ & $(g / d)$ & $(\mathrm{g} / \mathrm{g})$ \\
\hline WSM1 & 0 & 0 & $124.0 \pm 1.3$ & $1805.1^{\mathrm{a}} \pm 91.6$ & $91.3^{\mathrm{a}} \pm 9.0$ & $48.03^{\mathrm{a}} \pm 4.9$ & $1.85 \pm 0.1$ \\
\hline WSM2 & 250 & 0 & $123.3 \pm 1.1$ & $1853.2^{\mathrm{a}} \pm 73.7$ & $94.4^{\mathrm{a}} \pm 5.2$ & $49.43^{\mathrm{a}} \pm 2.0$ & $1.85 \pm 0.0$ \\
\hline WSM3 & 500 & 0 & $124.0 \pm 0.8$ & $1855.3^{\mathrm{a}} \pm 80.6$ & $95.7^{\mathrm{a}} \pm 8.4$ & $49.47^{\mathrm{a}} \pm 4.2$ & $1.88 \pm 0.0$ \\
\hline WSM4 & 0 & 1.5 & $124.3 \pm 1.7$ & $2255.6^{\mathrm{b}} \pm 54.0$ & $117.7^{\mathrm{b}} \pm 8.8$ & $60.89^{b} \pm 2.9$ & $1.85 \pm 0.1$ \\
\hline
\end{tabular}

${ }^{\mathrm{a}, \mathrm{b}}$ Means within the same column with different superscript letters are significantly different $(p<0.05)$.

mortality rate declined significantly (CSM3 vs. CSM1) but tended to be lowest following the positive control diet (CSM4). Growth data due to diet CSM3 were significantly below diet CSM4, indicating that the dietary $\mathbf{P}$ supply (CSM3) is still a limiting factor. Otherwise, it is not conclusive if the reachable effect of adding phytase is achieved with diet CSM3. Corresponding dietary $\mathbf{P}$ supply in WSM diets (Table 2) also yielded impaired zootechnical data (WSM1), but not so pronounced (Table 4). However, mortality rate was similar to diet CSM1 and decreased significantly by phytase supplementation (WSM 3). According to CSM diets, the iP supplementation (WSM4) significantly improved growth, feed intake, feed efficiency and mortality rate.

\section{Nutrient Deposition and Nutrient Utilization}

Growth data and nutrient analyses of body composition yielded the database for calculation of nutrient deposition and nutrient utilization (Table 5, Table 6). Nutrient deposition data were in line with the observed significant changes in growth performance. Phytase addition and iP supplementation in CSM diets yielded significantly improved deposition of crude protein, fat, ash, phosphorus and energy (Table 5). According to observed FCR data, the efficiency of protein and energy utilization was not significantly affected in CSM diets. In WSM diets (Table 6), phytase supplementation significantly improved crude protein deposition, but effects between WSM2 and WSM3 differed not significantly. Enhanced native phytase activity and consequently increased total phytase activity in WSM diets (Table 2) was indicated as a factor of infl- uence. However, due to iP supplementation (WSM4) further significant response was observed on crude ash, $\mathrm{P}$ and energy deposition, respectively. Diet WSM4 also yielded improved protein utilization.

\section{Tibia Composition}

The tibia analyses (Table 7), only recorded for experiment 1 (CSM diets), demonstrated significantly improved tibia ash contents due to supplementation of microbial phytase (CSM2, CSM3) and iP (CSM4), respectively. Between phytase addition and iP supplementation, the tibia ash content differed not significantly. Tibia content of $\mathrm{P}$ and $\mathrm{Ca}$ was significantly improved by addition of microbial phytase, but evidently, iP supplementation further improved $\mathrm{P}$ and $\mathrm{Ca}$ content of tibia dry matter.

\section{$N$ Balance Data}

Results with CSM diets indicated significantly improved daily $\mathrm{N}$ balance data following diet CSM3 (Table 8). $\mathrm{N}$ digestibility and $\mathrm{N}$ utilization responded in similar manner. Following diet CSM2, $\mathrm{N}$ digestibility only tended to be improved. Significant effects on $\mathrm{N}$ utilization were not observed both following iP supplementation (CSM4) and in WSM diets, respectively.

\section{$P$ Balance Data}

In CSM diets, phytase supplementation at $500 \mathrm{U} / \mathrm{kg}$ (CSM3) significantly improved daily $\mathrm{P}$ balance and $\mathrm{P}$ utilization, respectively (Table 9 ). Observed $\mathrm{P}$ balance data between diets CSM3 and CSM4 differed not significantly. In WSM diets, iP supplementation provided further significant increase of daily $\mathbf{P}$ balance, but $\mathbf{P}$ utilization remained similar to diet WSM1. Supplementa- 
Table 5. Nutrient deposition and nutrient utilization of chicken fed low phosphorus CSM diets (7-42 d) supplemented with microbial phytase or inorganic phosphorus and results of ANOVA, Experiment $1(n=6)$

\begin{tabular}{|c|c|c|c|c|c|c|c|c|c|c|}
\hline \multirow{3}{*}{ Diets } & \multirow{2}{*}{\multicolumn{2}{|c|}{ Supplementation }} & \multirow{3}{*}{$\begin{array}{l}\text { Crude } \\
\text { protein }\end{array}$} & \multicolumn{3}{|c|}{ Deposition } & \multirow{3}{*}{ Energy } & \multicolumn{3}{|c|}{ Utilization } \\
\hline & & & & \multirow{2}{*}{ Crude fat } & \multirow{2}{*}{ Crude ash } & \multirow{2}{*}{$\mathrm{P}$} & & \multirow{2}{*}{ Protein } & \multirow{2}{*}{$\mathbf{P}$} & \multirow{2}{*}{ Energy } \\
\hline & Phytase & iP & & & & & & & & \\
\hline & $(\mathrm{U} / \mathrm{kg})$ & $(\mathrm{g} / \mathrm{kg})$ & $(g / d)$ & $(g / d)$ & $(g / d)$ & $(g / d)$ & $(\mathrm{kJ} / \mathrm{d})$ & & $(\%)$ & \\
\hline CSM1 & 0 & 0 & $6.24^{\mathrm{a}} \pm 0.4$ & $4.06^{\mathrm{a}} \pm 1.1$ & $0.81^{\mathrm{a}} \pm 0.0$ & $0.12^{\mathrm{a}} \pm 0.0$ & $312^{\mathrm{a}} \pm 52$ & $43.1 \pm 3.0$ & $39.6^{\mathrm{a}} \pm 1.9$ & $35.9 \pm 5.8$ \\
\hline CSM2 & 250 & 0 & $7.60^{b} \pm 0.6$ & $5.25^{\mathrm{b}} \pm 1.1$ & $1.01^{\mathrm{b}} \pm 0.1$ & $0.16^{\mathrm{b}} \pm 0.0$ & $392^{b} \pm 49$ & $45.4 \pm 3.9$ & $46.4^{\mathrm{b}} \pm 4.3$ & $39.1 \pm 5.4$ \\
\hline CSM3 & 500 & 0 & $8.63^{c} \pm 0.5$ & $6.18^{\mathrm{b}} \pm 0.9$ & $1.24^{\mathrm{c}} \pm 0.1$ & $0.21^{\mathrm{c}} \pm 0.0$ & $454^{c} \pm 43$ & $45.3 \pm 1.9$ & $52.6^{\mathrm{c}} \pm 2.5$ & $39.7 \pm 2.5$ \\
\hline CSM4 & 0 & 1.5 & $10.48^{\mathrm{d}} \pm 0.3$ & $8.12^{c} \pm 0.5$ & $1.53^{\mathrm{d}} \pm 0.1$ & $0.25^{\mathrm{d}} \pm 0.0$ & $575^{\mathrm{d}} \pm 21$ & $44.4 \pm 4.9$ & $44.4^{\mathrm{ab}} \pm 7.1$ & $40.7 \pm 4.3$ \\
\hline
\end{tabular}

${ }^{\mathrm{a}, \mathrm{b}}$ Means within the same column with different superscript letters are significantly different $(p<0.05)$.

Table 6. Nutrient deposition and nutrient utilization of chicken fed low phosphorus WSM diets (7-42 d) supplemented with microbial phytase or inorganic phosphorus and results of ANOVA, Experiment $2(n=6)$

\begin{tabular}{|c|c|c|c|c|c|c|c|c|c|c|}
\hline \multirow{3}{*}{ Diets } & \multirow{2}{*}{\multicolumn{2}{|c|}{ Supplementation }} & \multirow{3}{*}{$\begin{array}{l}\text { Crude } \\
\text { protein }\end{array}$} & \multirow{3}{*}{ Crude fat } & \multicolumn{3}{|l|}{ Deposition } & \multicolumn{3}{|c|}{ Utilization } \\
\hline & & & & & \multirow{2}{*}{ Crude ash } & \multirow{2}{*}{$\mathrm{P}$} & \multirow{2}{*}{ Energy } & \multirow{2}{*}{ Protein } & \multirow{2}{*}{$\mathrm{P}$} & \multirow{2}{*}{ Energy } \\
\hline & Phytase & iP & & & & & & & & \\
\hline & $(\mathrm{U} / \mathrm{kg})$ & $(\mathrm{g} / \mathrm{kg})$ & $(g / d)$ & $(g / d)$ & $(g / d)$ & $(g / d)$ & $(\mathrm{kJ} / \mathrm{d})$ & & $(\%)$ & \\
\hline WSM1 & 0 & 0 & $6.88^{\mathrm{a}} \pm 1.1$ & $5.35 \pm 1.8$ & $1.08^{\mathrm{a}} \pm 0.2$ & $0.19^{\mathrm{a}} \pm 0.0$ & $379^{a} \pm 91$ & $37.7^{\mathrm{a}} \pm 3.0$ & $54.2^{\mathrm{ab}} \pm 5.9$ & $33.7 \pm 6.3$ \\
\hline WSM2 & 250 & 0 & $8.51^{\mathrm{b}} \pm 0.7$ & $5.31 \pm 0.9$ & $1.19^{\mathrm{a}} \pm 0.1$ & $0.20^{\mathrm{a}} \pm 0.0$ & $417^{a} \pm 25$ & $45.3^{\mathrm{ab}} \pm 4.0$ & $56.1^{\mathrm{ab}} \pm 3.9$ & $36.1 \pm 1.8$ \\
\hline WSM3 & 500 & 0 & $8.50^{\mathrm{b}} \pm 1.0$ & $5.04 \pm 0.9$ & $1.23^{\mathrm{a}} \pm 0.1$ & $0.22^{\mathrm{a}} \pm 0.0$ & $403^{\mathrm{a}} \pm 40$ & $44.2^{\mathrm{ab}} \pm 3.9$ & $61.4^{\mathrm{b}} \pm 5.4$ & $34.4 \pm 1.6$ \\
\hline WSM4 & 0 & 1.5 & $10.69^{c} \pm 2.1$ & $6.72 \pm 1.9$ & $1.59^{\mathrm{b}} \pm 0.4$ & $0.29^{\mathrm{b}} \pm 0.1$ & $525^{\mathrm{b}} \pm 122$ & $46.0^{\mathrm{b}} \pm 10.2$ & $46.4^{\mathrm{a}} \pm 10.4$ & $36.9 \pm 9.7$ \\
\hline
\end{tabular}

${ }^{\mathrm{a}, \mathrm{b}}$ Means within the same column with different superscript letters are significantly different $(p<0.05)$.

Table 7. Tibia composition ( $\%$ of DM) of chicken fed low phosphorus CSM diets (7-42 d) supplemented with microbial phytase or inorganic phosphorus and results of ANOVA, Experiment $1(n=6)$

\begin{tabular}{|c|c|c|c|c|}
\hline Diets & CSM1 & CSM2 & CSM3 & CSM4 \\
\hline Supplementation & 0 & $250 \mathrm{U} / \mathrm{kg}$ & $500 \mathrm{U} / \mathrm{kg}$ & $1.5 \mathrm{giP} / \mathrm{kg}$ \\
\hline Tibia ash $(\mathrm{DM} \%)$ & $35.4^{\mathrm{a}} \pm 1.7$ & $39.4^{\mathrm{b}} \pm 3.4$ & $40.0^{\mathrm{b}} \pm 1.7$ & $41.9^{\mathrm{b}} \pm 1.6$ \\
\hline $\mathrm{P}$ in tibia $(\mathrm{DM} \%)$ & $5.6^{a} \pm 0.3$ & $6.3^{\mathrm{b}} \pm 0.5$ & $6.6^{\mathrm{bc}} \pm 0.4$ & $7.0^{c} \pm 0.3$ \\
\hline $\mathrm{Ca}$ in tibia $(\mathrm{DM} \%)$ & $12.7^{\mathrm{a}} \pm 1.0$ & $14.6^{\mathrm{b}} \pm 1.4$ & $14.5^{\mathrm{b}} \pm 0.8$ & $15.2^{\mathrm{b}} \pm 0.8$ \\
\hline
\end{tabular}

tion of $500 \mathrm{U} / \mathrm{kg}$ microbial phytase (CSM3, WSM3) improved $\mathbf{P}$ utilization significantly. The basal diet was a main factor of influence on $\mathbf{P}$ balance data including $\mathbf{P}$ utilization.

\section{Effects on $A M E$}

Determination of metabolizable energy (AMEn) content in the CSM and WSM diets did not yield significant treatment effects (Table 10). Consequently, established AMEn content of the diets under study did not indicate significant effects of added phytase on availability of dietary energy.

\section{Discussion}

Following low phosphorus CSM diets, feed intake and growth data responded significantly due to graded supply of microbial phytase (Yi et al., 1996a; Qian et al., 1997). This observation is in line with many reports (Schöner et al., 1991; Kornegay et al., 1996; Rama Rao et al., 1999; Zhang et al., 2000; Lan et al., 2002). Earlier studies using CSM diets also observed strong effects of low $\mathrm{P}$ diets on zootechnical data (Sebastian et al., 1996, 1997; Rama Rao et al., 1999; Pillai et al., 2002) and mortality rate (Simons et al., 1990; Denbow et al., 1995; Sohail and Roland, 1999). Supplementation of inorganic $P$ yielded further improved zoo technical data, indicating that the enhanced $\mathrm{P}$ availability by added phytase was not equal to $\mathrm{P}$ supply following iP supplementation. Significant dose response effects due to added phytase lead to speculation that 
Table 8. $\mathrm{N}$ balance data $\left(\mathrm{mg} / \mathrm{BW}_{\mathrm{kg}}{ }^{0.67} / \mathrm{d}\right)$ of chicken fed low phosphorus CSM and WSM diets (factor basal diet) supplemented with microbial phytase or inorganic phosphorus $(n=8)$ and results $(p)$ of univariate ANOVA, Experiment $1+2$

\begin{tabular}{|c|c|c|c|c|c|c|c|c|}
\hline Diets & CSM1 & CSM2 & CSM3 & CSM4 & WSM1 & WSM2 & WSM3 & WSM4 \\
\hline Supplementation & 0 & $250 \mathrm{U} / \mathrm{kg}$ & $500 \mathrm{U} / \mathrm{kg}$ & $1.5 \mathrm{~g} \mathrm{iP} / \mathrm{kg}$ & 0 & $250 \mathrm{U} / \mathrm{kg}$ & $500 \mathrm{U} / \mathrm{kg}$ & $1.5 \mathrm{~g} \mathrm{iP} / \mathrm{kg}$ \\
\hline $\mathrm{N}$ intake & $3587 \pm 184$ & $3571 \pm 266$ & $3605 \pm 144$ & $3576 \pm 90$ & $3589 \pm 103$ & $3602 \pm 142$ & $3505 \pm 87$ & $3528 \pm 152$ \\
\hline $\mathrm{N}$ excretion & $1678^{a} \pm 123$ & $1539^{\mathrm{ab}} \pm 220$ & $1484^{\mathrm{b}} \pm 84$ & $1674^{a} \pm 78$ & $1704^{\mathrm{a}} \pm 123$ & $1616^{\mathrm{a}} \pm 175$ & $1587^{a} \pm 44$ & $1618^{a} \pm 112$ \\
\hline $\mathrm{N}$ balance & $1909^{a} \pm 111$ & $2032^{\mathrm{ab}} \pm 195$ & $2122^{b} \pm 123$ & $1903^{\mathrm{a}} \pm 136$ & $1885^{a} \pm 125$ & $1986^{a} \pm 167$ & $1918^{a} \pm 91$ & $1910^{a} \pm 121$ \\
\hline $\mathrm{N}$ digestibility (\%) & $80.6^{a} \pm 1.9$ & $81.0^{\mathrm{a}} \pm 1.2$ & $83.2^{\mathrm{b}} \pm 0.9$ & $82.8^{\mathrm{b}} \pm 1.6$ & $81.8^{\mathrm{a}} \pm 2.5$ & $82.5^{\mathrm{a}} \pm 1.9$ & $81.6^{\mathrm{a}} \pm 0.7$ & $81.8^{\mathrm{a}} \pm 1.8$ \\
\hline $\mathrm{N}$ utilization $(\%)$ & $53.2^{\mathrm{a}} \pm 2.1$ & $57.0^{\mathrm{b}} \pm 4.6$ & $58.8^{\mathrm{b}} \pm 2.0$ & $53.2^{\mathrm{a}} \pm 2.8$ & $52.3^{\mathrm{a}} \pm 3.2$ & $55.2^{\mathrm{a}} \pm 4.3$ & $54.7^{\mathrm{a}} \pm 1.6$ & $54.2^{\mathrm{a}} \pm 2.5$ \\
\hline
\end{tabular}

\begin{tabular}{lccccc}
\hline \multirow{2}{*}{ Factor } & \multicolumn{5}{c}{$p$-value } \\
\cline { 2 - 6 } & Basal diet & Phytase & iP & Basal diet x Phytase & Basal diet x iP \\
\hline N intake & 0.407 & 0.811 & 0.526 & 0.480 & 0.655 \\
N excretion & 0.398 & $\mathbf{0 . 0 0 5}$ & 0.347 & 0.714 & 0.389 \\
N balance & 0.104 & $\mathbf{0 . 0 3 6}$ & 0.857 & 0.146 & 0.088 \\
N digestibility (\%) & 0.572 & 0.138 & 0.074 & $\mathbf{0 . 0 2 7}$ & 0.440 \\
N utilization $(\%)$ & 0.123 & $\mathbf{0 . 0 0 2}$ & 0.484 & 0.297 & \\
\hline
\end{tabular}

${ }^{\mathrm{a}, \mathrm{b}}$ Means within the same line (based on statistical analysis within the respective balance period) with different superscript letters are significantly different $(p<0.05)$.

Table 9. $\mathbf{P}$ balance data $\left(\mathrm{mg} / \mathrm{LW}_{\mathrm{kg}}{ }^{0.67} / \mathrm{d}\right)$ of chicken fed low phosphorus CSM and WSM diets (factor basal diet) supplemented with microbial phytase or inorganic phosphorus $(n=8)$ and results $(p)$ of univariate ANOVA, Experiment $1+2$

\begin{tabular}{|c|c|c|c|c|c|c|c|c|}
\hline Diets & CSM1 & CSM2 & CSM3 & CSM4 & WSM1 & WSM2 & WSM3 & WSM4 \\
\hline Supplementation & 0 & $250 \mathrm{U} / \mathrm{kg}$ & $500 \mathrm{U} / \mathrm{kg}$ & $1.5 \mathrm{~g} \mathrm{iP} / \mathrm{kg}$ & 0 & $250 \mathrm{U} / \mathrm{kg}$ & $500 \mathrm{U} / \mathrm{kg}$ & $1.5 \mathrm{~g} \mathrm{iP} / \mathrm{kg}$ \\
\hline$P$ intake & $462^{\mathrm{a}} \pm 25$ & $460^{\mathrm{a}} \pm 34$ & $463^{a} \pm 18$ & $538^{\mathrm{b}} \pm 13$ & $436^{\mathrm{b}} \pm 12$ & $418^{\mathrm{ab}} \pm 16$ & $408^{a} \pm 10$ & $579^{c} \pm 25$ \\
\hline $\mathbf{P}$ excretion & $253^{\mathrm{a}} \pm 28$ & $224^{\mathrm{a}} \pm 45$ & $220^{a} \pm 35$ & $304^{\mathrm{b}} \pm 26$ & $146^{\mathrm{b}} \pm 26$ & $122^{\mathrm{ab}} \pm 19$ & $110^{\mathrm{a}} \pm 18$ & $217^{c} \pm 47$ \\
\hline $\mathrm{P}$ balance & $209^{\mathrm{a}} \pm 11$ & $236^{\mathrm{ab}} \pm 33$ & $243^{b} \pm 37$ & $234^{\mathrm{ab}} \pm 25$ & $289^{a} \pm 31$ & $296^{\mathrm{a}} \pm 24$ & $298^{a} \pm 12$ & $362^{\mathrm{b}} \pm 37$ \\
\hline \multirow[t]{2}{*}{ P utilization $(\%)$} & $45.3^{\mathrm{ab}} \pm 3.7$ & $51.6^{\mathrm{bc}} \pm 8.2$ & $52.4^{c} \pm 7.8$ & $43.5^{\mathrm{a}} \pm 4.6$ & $66.3^{\mathrm{ab}} \pm 6.3$ & $70.8^{\mathrm{bc}} \pm 4.7$ & $73.1^{c} \pm 3.9$ & $62.6^{\mathrm{a}} \pm 7.2$ \\
\hline & \multicolumn{8}{|c|}{$p$-value } \\
\hline Factor & Basal diet & Phytase & iP & \multicolumn{2}{|c|}{ Basal diet $\times$ Phytase } & \multicolumn{2}{|c|}{ Basal diet $\times$ iP } & \\
\hline$P$ intake & 0.015 & 0.201 & $<0.0001$ & \multicolumn{2}{|c|}{0.160} & \multicolumn{2}{|c|}{$<0.0001$} & \\
\hline $\mathrm{P}$ excretion & $<0.0001$ & 0.012 & $<0.0001$ & \multicolumn{2}{|c|}{0.933} & \multicolumn{2}{|c|}{0.397} & \\
\hline $\mathrm{P}$ balance & $<0.0001$ & 0.044 & $<0.0001$ & \multicolumn{2}{|c|}{0.626} & \multicolumn{2}{|c|}{0.033} & \\
\hline $\mathrm{P}$ utilization $(\%)$ & $<0.0001$ & 0.007 & 0.210 & \multicolumn{2}{|c|}{0.903} & \multicolumn{2}{|c|}{0.644} & \\
\hline
\end{tabular}

${ }^{\mathrm{a}, \mathrm{b}}$ Means within the same line (based on statistical analysis within the respective balance period) with different superscript letters are significantly different $(p<0.05)$.

Table 10. $\mathbf{A M E}_{\mathrm{n}}$ of low phosphorus CSM and WSM chicken diets ${ }^{1)}$ supplemented with microbial phytase or inorganic phosphorus $(\mathrm{n}=8)$, Experiment $1+2$

\begin{tabular}{ccccccccc}
\hline \hline Diets & CSM1 & CSM2 & CSM3 & CSM4 & WSM1 & WSM2 & WSM3 & WSM4 \\
Supplementation & 0 & $250 \mathrm{U} / \mathrm{kg}$ & $500 \mathrm{U} / \mathrm{kg}$ & $1.5 \mathrm{~g} \mathrm{iP} / \mathrm{kg}$ & 0 & $250 \mathrm{U} / \mathrm{kg}$ & $500 \mathrm{U} / \mathrm{kg}$ & $1.5 \mathrm{~g} \mathrm{iP} / \mathrm{kg}$ \\
\hline $\mathrm{AME}_{\mathrm{n}}(\mathrm{MJ} / \mathrm{kg})$ & $13.25 \pm 0.13$ & $13.38 \pm 0.40$ & $13.74 \pm 0.42$ & $13.74 \pm 0.24$ & $13.39 \pm 0.40$ & $13.58 \pm 0.25$ & $13.35 \pm 0.18$ & $13.39 \pm 0.25$ \\
\hline
\end{tabular}

\footnotetext{
${ }^{1)}$ Results of univariate ANOVA for all diets provided $p>0.05$.
}

further increments of supplementation could provide additional effects on nutrient deposition. In contrast to several observations (Kornegay et al., 1997; Zhang et al., 2000; Lan et al., 2002), no significant effect on FCR was observed. Following CSM diets, FCR tended to decline according to phytase addition. However, WSM diets with added phytase did not provide a similar trend due to the enhanced native phytase activity in these diets.

Following low phosphorus WSM diets, according to earlier reports (Huyghebaert, 1996; Ravindran et al., 1999 
a, b) the supplementation of microbial phytase did not improve growth data significantly. This observation was not in agreement with Zyla et al. (2000). However, iP supplementation further improved growth data significantly, indicating that the achieved $\mathrm{P}$ supply from WSM diets was still below the requirement of growing chicken.

$\mathbf{P}$ deposition established from body analyses was significantly increased by phytase addition to CSM diets. According to Schöner et al. (1991), the P utilization was significantly enhanced by microbial phytase. Following the WSM diets, these effects were observed only numerical, indicating that the phytase accessible part of the phytate phosphorus in WSM diets becomes mostly available by the native phytase activity. Consequently, graded supply of microbial phytase only tended to improve $\mathbf{P}$ deposition. Protein and energy deposition responded in similar manner. Protein and energy utilization based on results of body analyses were not significantly improved by supplementation of microbial phytase addition in CSM and WSM diets, respectively. The observed trends were more pronounced in the balance studies. In CSM diets, the protein utilization significantly responded to phytase supplementation. Surprisingly, this effect was not observed following WSM diets with enhanced native phytase activity coming from basal diet. Significant phytase effects on $\mathrm{N}$ utilization were observed in several studies (Yi et al., 1994, 1996a, b; Kornegay et al., 1996), indicating the occurrence of phytase accessible phytate-protein-complexes during digestion (Farrell et al., 1993). Otherwise, direct phytate effects on digestive enzyme activity (Singh and Krikorian, 1982; Deshpande and Cheryan, 1984) and endogenous amino acid flow (Cowieson and Ravindran, 2007) were reported. Consequently, enhanced N utilization also could be the result of decreased endogenous amino acid losses. Established main effects from univariate ANOVA realize $\mathrm{N}$ balance, $\mathrm{N}$ excretion and $\mathrm{N}$ utilization as significantly influenced by phytase addition. The observed effects on $\mathbf{N}$ excretion in CSM diets were according to Yi et al. (1996c). Additionally, for $\mathrm{N}$ digestibility an interrelationship was observed between basal diet and phytase addition. This observation is supporting the growth data, which yielded a tendency of improved protein utilization only due to supplementation of microbial phytase.

$\mathrm{P}$ utilization derived from balance studies was influenced by the basal diet, indicating the importance of the native phytase activity. Additionally, the supplemented microbial phytase provided main effects on $\mathbf{P}$ excretion, $\mathrm{P}$ balance and $\mathbf{P}$ utilization, respectively. As expected, iP supplementation yielded main effects on $\mathrm{P}$ balance data. However, iP addition was not a main factor for the observed $\mathrm{P}$ utilization. According to Viveros et al. (2002), adding iP decreased $\mathbf{P}$ utilization. Additionally, $\mathbf{P}$ utilization data concluded from long-term deposition studies based on analysis of body composition and shortterm $\mathbf{P}$ balance experiment are not directly comparable. Due to the prolonged exhausting of $\mathbf{P}$ depots during the long-term study, suboptimal $\mathrm{P}$ supply provided effects that are more pronounced. Furthermore, interrelationships between dietary $\mathrm{Ca}$ supply and phytate degradation has to be taken into account. Reports in growing chicken (Plumstead et al., 2008; Leytem et al., 2008) indicate that our current investigations worked on $\mathrm{Ca}$ to NPP ratio where the efficiency of phytate hydrolysis could be partly depressed by dietary Ca supply. However, this statement is not free of speculation and verification is needed. Finally, caloric measurements in feed and excreta within the balance studies yielded no evidence of significant improvement of dietary AME by phytase addition, as reported in several studies (Ravindran et al., 1999b, 2001).

In conclusion, current results with supplemented phytase in low phosphorus chicken CSM and WSM diets observed significant improvement of $\mathrm{P}$ utilization in both diets. The basal diet was a significant factor for the established level of $\mathbf{P}$ utilization. Phytase addition was also a main factor for $\mathrm{N}$ utilization, but only in CSM diets with marginal native phytase activity the $\mathrm{N}$ utilization responded significantly. The observed response was only partly explained by improved protein digestibility and this contrast needs further investigations to provide reliable conclusions due to conditions under which a significant effect of added phytase on $\mathrm{N}$ utilization can be expected. Finally, the added phytase source could be a further factor of influence on observed nutrient utilization as reported by Liebert and Portz (2005, 2007).

\section{Acknowledgments}

The authors express their thanks to the former Roche Vitamins Ltd. Company for supplying the experimental phytase, analyses of in-feed phytase activity and financial support of the experiments.

\section{References}

Association of Official Analytical Chemists. Method No. 986.11. Official Methods of Analysis. $15^{\text {th }}$ Ed. Association of Official Analytical Chemists, Washington, DC. 1990.

Augspurger NR and Baker DH. High dietary phytase levels maximize phytate-phosphorus utilization but do not affect protein utilization in chicks fed phosphorus- or amino aciddeficient diets. Journal Animal Science, 82: 1100-1107. 2004.

Augspurger NR, Webel DM, Lei XG and Baker DH. Efficacy of an E. coli phytase expressed in yeast for releasing phytatebound phosphorus in young chicks and pigs. Journal Animal Science, 81: 474-483. 2003.

Bedford MR. Exogenous enzymes in monogastric nutrition their current value and future benefits. Journal of Animal Feed Science and Technology, 86: 1-13. 2000.

Boling SD, Douglas MW, Johnson ML, Wang X, Parsons CW, Koelkebeck KW and Zimmerman RA. Supplemental phytase improves performance of laying hens consuming diets with low levels of available phosphorus. Poultry Science, 76 (Suppl. 1): 20. (Abstr.). 1997.

Boling SD, Douglas MW, Shirley RB, Parsons CW and Koelkebeck KW. The effects of various dietary levels of phytase and available phosphorus on performance of laying 
hens. Poultry Science, 79: 535-538. 2000a.

Boling SD, Douglas MW, Johnson ML, Wang X, Parsons CW, Koelkebeck KW and Zimmerman RA. The effects of dietary available phosphorus levels and phytase on performance of young and older laying hens. Poultry Science, 79: 224-225. 2000b.

Caldwell RA. Effect of calcium and phytic acid on the activation of trypsinogen and the stability of trypsin. Journal of Agricultural and Food Chemistry, 40: 43-47. 1992.

Carlos AB and Edwards HM, Jr. The effects of 1,25dihydroxycholecalciferol and phytase on the natural phytate phosphorus utilization by laying hens. Poultry Science, 77: 850-858. 1998.

Ceylan N, Scheideler SE and Stilborn HL. High available phosphorus corn and phytase in layer diets. Poultry Science, 82: 789-795. 2003.

Cowieson AJ and Ravindran V. Effect of phytic acid and microbial phytase on the flow and amino acid composition of endogenous protein at the terminal ileum of growing broiler chicken. British Journal of Nutrition, 98: 745-752. 2007

Davies MI, Ritcey GM and Motzok I. Intestinal phytase and alkaline phosphatise of chick: Influence of dietary calcium, inorganic and phytate phosphorus and vitamin $\mathrm{D}_{3}$. Poultry Science, 49: 1280-1285. 1970.

Denbow DM, Ravindram V, Kornegay ET, Yi Z and Hulet RM. Improving phosphorus availability in soybean meal for broilers by supplemented phytase. Poultry Science, 74: 18311842. 1995.

Deshpande SS and Cheryan M. Effects of phytate, divalent cations and their implications on alpha-amylase activity. Journal of Food Science, 49: 516. 1984.

Ebrahimnezhad Y, Shivazad M, Taherkhani R and Nazeradl K. Effects of citric acid and microbial phytase supplementation on performance and phytase phosphorus utilization in broiler chicks. The Journal of Poultry Science, 45: 20-24. 2008.

Engelen AJ, Van der Heeft FC, Randsdorp PH and Smit EL. Simple and rapid determination of phytase activity. Journal of AOAC International, 77: 760-764. 1994.

Farrell DJ, Martin EA, du Preez J, Bongarts M, Betts M, Sudaman A and Thomson E. The beneficial effects of a microbial phytase in diets of broiler chickens and ducklings. Journal of Animal Physiology and Animal Nutrition, 69: 278-283. 1993.

Gifford SR and Clydesdale FM. Interactions among calcium, zinc and phytate with three protein sources. Journal of Food Science, 55: 1720-1724. 1990.

Gordon RW and Roland DA. Performance of commercial laying hens fed various phosphorus levels with and without supplemental phytase. Poultry Science, 76: 1172-1177. 1997.

Gordon RW and Roland DA, Sr. Influence of supplemental phytase on calcium and phosphorus utilization in laying hens. Poultry Science, 77: 290-294. 1998.

Huyghebaert G. Effects of dietary calcium, phosphorus, Ca/ P-ration and phytase on zootechnical performances and mineralization in broiler chicks. Archiv für Geflügelkunde, 61 (2): 53-61. 1996.

Jalal MA and Scheideler SE. Effect of supplementation of two different sources of phytase on egg production parameters in laying hens and nutrient digestibility. Poultry Science, 80: 1463-1471. 2001.

Juanpere J, Perez-Vendrell AM and Brufau J. Effect of microbial phytase on broilers fed barley-based diets in the presence or not of endogenous phytase. Journal Animal Feed Science and Technology, 115: 265-279. 2004.

Keshavarz K. Effects of continuous feeding of low-phosphorus diets with and without phytase during the growing and laying periods on performance of two strains of leghorns. Poultry Science, 82: 1444-1456. 2003a.

Keshavarz K. The effect of different levels of nonphytate phosphorus with and without phytase on the performance of four strains of laying hens. Poultry Science 82: 71-91. 2003b.

Knuckles BE, Kuzmicky DD, Gumbmann MR and Betschart AA. Effect of myo-inositol phosphate esters on in vivo digestion of protein. Journal of Food Science, 54: 13481350. 1989.

Kornegay ET, Denbow DM, Yi Z and Ravindran V. Response of broilers to graded levels of microbial phytase added to maize-soybean meal-based diets containing three levels of non-phytate phosphorus. British Journal of Nutrition, 75: 839-852. 1996.

Kornegay ET, Denbow DM and Zhang Z. Phytase supplementation of corn-soybean meal broiler diets from three to seven week. Poultry Science, 76 (Suppl. I): 6 (Abstr.). 1997.

Kornegay ET. Digestion of phosphorus and other nutrients: the role of phytases and factors influencing their activity. In: Enzymes in Farm Animal Nutrition. (Bedford MR and Partridge GG eds.). pp. 237-259. 2001.

Lan GQ, Abdullah N, Jalaludin S and Ho YW. Efficacy of supplementation of a phytase-producing bacterial culture on the performance and nutrient use of broiler chickens fed corn-soybean meal diets. Poultry Science, 81: 1522. 2002.

Leytem AB, Plumstead PW, Maguire RO, Kwanyuen P, Burton JW and Brake J. Interaction of calcium and phytate in broiler diets. 2. Effects on total and soluble phosphorus excretion. Poultry Science, 87: 459-467. 2008.

Liebert $F$ and Portz L. Nutrient utilization of Nile tilapia Oreochromis niloticus fed plant based low phosphorus diets supplemented with graded levels of different sources of microbial phytase. Aquaculture, 248: 111-119. 2005.

Liebert F, Htoo JK and Sünder A. Performance and nutrient utilization of laying hens fed low-phosphorus corn-soybean and wheat-soybean diets supplemented with microbial phytase. Poultry Science, 84: 1576-1583. 2005.

Liebert F and Portz L. Different sources of microbial phytase in plant based low phosphorus diets for Nile tilapia Oreochromis niloticus may provide different effects on phytate degradation. Aquaculture, 267: 292-299. 2007.

Lim HS, Namkung $H$ and Paik IK. Effects of phytase supplementation on the performance, egg quality, and phosphorus excretion of laying hens fed different levels of dietary calcium and nonphytate phosphorus. Poultry Science, 82: 92-99. 2003.

Namkung $\mathrm{H}$ and Leeson S. Effect of phytase enzyme on dietary nitrogen-corrected apparent metabolizable energy and the ileal digestibility of nitrogen and amino acids in broiler chicks. Poultry Science, 78: 1317-1319. 1999.

National Research Council. Nutrient requirements of poultry. $9^{\text {th }}$ rev. ed. National Academy Press, Washington, DC. 1994.

Naumann K and Bassler R. Die chemische Untersuchung von Futtermitteln. Methodenbuch, Bd. III., Verlag NeumannNeudam. 1976-1997.

Nelson TS. The utilization of phytate phosphorus by poultry. A Review. Poultry Science, 46: 862-869. 1967.

O'Dell BL and De Boland A. Complexation of phytate with 
proteins and cations in corn germ and oilseed meals. Journal of Agricultural and Food Chemistry, 24: 804-808. 1976.

Onyango EM, Bedford MR and Adeola O. Efficacy of an evolved Escherischia coli phytase in diets of broiler chicks. Poultry Science, 84: 248-255. 2005.

Pahle T, Köhler R, Halle I, Jeroch H and Gebhardt G. Methodische Untersuchungen zur Bestimmung der Verdaulichkeit des Rohproteins beim Hühnergeflügel. Archive of Animal Nutrition, 33: 363-370. 1983.

Pahle T, Köhler R, Halle I, Jeroch $\mathrm{H}$ and Gebhardt G. Die Bestimmung der Rohproteinverdaulichkeit beim Hühnergeflügel mit der $\alpha-\mathrm{NH}_{2}-\mathrm{N}-$ Methode. Archive of Animal Nutrition, 35: 82-87. 1985.

Payne RL, Lavergne TK and Southern LL. A comparison of two sources of phytase in liquid and dry forms in broilers. Poultry Science, 84: 265-272. 2005.

Peter W, Zachmann R and Jeroch H. Zum Phytaseeinsatz bei Legehennen. in: Proceedings Internationale Tagung Schweine- und Geflügelernährung, Halle, Germany. pp. 7883. 1992.

Peter CM, Han Y, Boling-Frankenbach SD, Parsons CM and Baker DH. Limiting order of amino acids and the effects of phytase on protein quality in corn gluten meal fed to young chicks. Journal of Animal Science, 78: 2150-2156. 2000.

Pillai PB, Emmert JL and Webel DM. Impact of phytex phytase on phytate-bound phosphorus release and growth performance of broiler chicks. Poultry Science Association. $\left(91^{\text {st }}\right.$ Annual Meeting Abstracts). Poscal 80 (Suppl. I): 76. 2002.

Plumstead PW, Leytem AB, Maguire RO, SpearsW, Kwanyuen $\mathbf{P}$ and Brake $\mathbf{J}$. Interaction of calcium and phytate in broiler diets. 1. Effects on apparent prececal digestibility and retention of phosphorus. Poultry Science, 87: 449-458. 2008.

Qian H, Kornegay ET and Denbow DM. Utilization of phytase phosphorus and calcium as influenced microbial phytase, cholecalciferol, and the calcium total phosphorus ration in broiler diets. Poultry Science, 75: 618-626. 1997.

Rama Rao SV, Ravindra Reddy V and Ramasubba Reddy V. Enhancement of phytate phosphorus availability in the diets of commercial broilers and layers. Journal of Animal Feed Science and Technology, 79: 211-222. 1999.

Ravindran V, Cabahug S, Ravindran G and Bryden WL. 1999a. Influence of microbial phytase on apparent ileal amino acid digestibility of feedstuffs for broilers. Poultry Science, 78: 699-706. 1999a.

Ravindran V, Selle PH and Bryden WL. Effect of phytase supplementation, individually and in combination, with glycanase, on the nutritive value of wheat and barley. Poultry Science, 78: 1588-1595. 1999b.

Ravindran V, Selle PH, Ravindran G, Morel PCH, Kies AK and Bryden WL. Microbial phytase improves performance, apparent metabolizable energy, and ileal amino acid digestibility of broilers fed a lysine-deficient diet. Poultry Science, 80: 338-344. 2001.

Reddy MK, Sathe SK and Pierson MD. Removal of phytate from great northern beans (Phaseolus vulgaris L.) and its combined density fractions. Journal of Food Science, 53: 107-110. 1988.

Rutherford SM, Chung TK, Morel PCH and Moughan PJ. Effect of microbial phytase on ileal digestibility of phytate phosphorus, total phosphorus, and amino acids I a lowphosphorus diet for broilers. Poultry Science, 83: 61-68. 2004.
Schöner FJ, Hoppe PP and Schwarz G. Comparative effects of microbial phytase and inorganic phosphorus on performance and retentions of phosphorus, calcium and crude ash in broilers. Journal of Animal Physiology and Animal Nutrition, 66: 248-225. 1991.

Sebastian S, Touchburn SP, Chaves ER and Lague PC. The effects of supplemental microbial phytase on the performance and utilization of dietary calcium, phosphorus, copper and zinc in broiler chickens fed corn-soybean diets. Poultry Science, 75: 729-736. 1996.

Sebastian S, Touchburn SP, Chaves ER and Lague PC. Apparent digestibility of protein and amino acids in broiler chickens fed a corn-soybean diet supplemented with microbial phytase. Poultry Science, 76: 1760-1769. 1997.

Shan AS, Wang LJ, Song JC, Wang A, Du J, Xu QY and Zhang ZY. Performance and nutrient utilization of layers fed diet supplemented with microbial phytase and cellulase. Poultry Science Association (91 ${ }^{\text {st }}$ Annual Meeting Abstracts), Poscal 80 (Suppl. 1): 138. 2002.

Shirley RB and Edwards HM, Jr. Dietary calcium affects phytase activity when phytase is supplemented in excess of industry standards. Poultry Science Association (91 ${ }^{\text {st }}$ Annual Meeting Abstracts). Poscal 80 (Suppl. 1): 11. 2002.

Simons PCM, Versteegh HAJ, Jongbloed AW, Kemme PA, Slump P, Bos KD, Wolters MGE, Beudeker RF and Verschoor GJ. Improvement of phosphorus availability by microbial phytase in broilers and pigs. British Journal of Nutrition, 64: 525-540. 1990.

Singh $\mathrm{M}$ and Krikorian AD. Inhibition of trypsin activity in vitro by phytate. Journal of Agricultural and Food Chemistry, 30: 799. 1982.

Snow JL, Baker DH and Parsons CM. Phytase, citric acid, and 1 $\alpha$-hydroxycholecalciferol improve phytate phosphorus utilization in chicks fed a corn-soybean meal diet. Poultry Science, 83: 1187-1192. 2004.

Sohail SS and Roland DA, Sr. Influence of supplemental phytase on performance of broilers four to six weeks of age. Poultry Science, 78: 50-555. 1999.

Titus HW, Mehring AL, Johnson D, Nesbitt LL and Tomas T. An evaluation of M.C.F. (Micro-Cel-Fat), a new type of fat product. Poultry Science, 38: 1114-1119. 1959.

Vaintraub IA and Bulmaga VP. Effect of phytate on the in vitro activity of digestive proteinases. Journal of Agricultural and Food Chemistry, 39: 859-861. 1991.

Van der Klis JD, Versteegh HAJ, Simons PCM and Kies AK. The efficacy of phytase in corn-soybean meal-based diets for laying hens. Poultry Science, 76: 1535-1542. 1997.

Viveros A, Brenes A, Arija I and Centeno C. Effects of microbial phytase supplementation on mineral utilization and serum enzyme activities in broiler chicks fed different levels of phosphorus. Poultry Science, 81: 1172-1183. 2002.

WPSA. World's Poultry Science Journal, 40: 181-182. 1984.

Yi Z, Kornegay ET, Ravindran V and Denbow DM. Corn and soybean meal $\mathrm{P}$ for broilers using Natuphos phytase and calculation of replacement values of inorganic phosphorus by phytase. Poultry Science, 73 (Suppl. I): 89. 1994.

Yi Z, Kornegay ET, Ravindran V and Denbow DM. Improving phytate phosphorus availability in corn and soybean meal for broilers using microbial phytase and calculation of phosphorus equivilancy for phytase. Poultry Science, 75: 240249. 1996a.

Yi Z, Kornegay ET and Denbow DM. Supplemental microbial 
phytase improves zinc utilization in broilers. Poultry Science, 75: 540-546. 1996b.

Yi Z, Kornegay ET, Ravindran V and Denbow DM. Effect of microbial phytase on nitrogen and amino acid digestibility and nitrogen retention of turkey poults fed corn-soybean meal diets. Poultry Science, 75: 979-990. 1996c.

Zhang S, Roland DA, McDaniel GR and Rao SK. Effect of Natuphos phytase supplementation to feed on performance and ileal digestibility of protein and amino acids of broilers. Poultry Science, 78: 1567-1572. 1999.

Zhang ZB, Kornegay ET, Radcliffe JS, Denbow DM, Veit HP and Larsen CT. Comparison of genetically engineered microbial and plant phytase for young broilers. Poultry Science, 79: 709-717. 2000.

Zyla K, Koreleski J, Swiatkiewicz S, Wikiera A, Kujawski M, Piironen J and Ledoux DR. Effect of phosphorolytic and cell wall-degrading enzymes on the performance of growing broilers fed wheat-based diets containing different calcium levels. Poultry Science, 79: 66-76. 2000.

Zyla K, Mika M, Stodolak B, Wikiera A, Koreleski J and Swiatkiewicz S. Towards complete dephosphorylation and total conversion of phytates in poultry feeds. Poultry Science, 83: 1175-1186. 2004. 